\title{
A P2P COLLABORATIVE FRAMEWORK FOR SMARTPHONE
}

\author{
Heien-Kun Chiang ${ }^{1}$, Feng-Lan Kuo ${ }^{2}$, Meng-Ting Chen ${ }^{1}$ \\ ${ }^{1}$ Information Management Department, National Changhua University of Education \\ hkchiang@cc. ncue .edu. tw \\ ${ }^{2}$ English Department \& Graduate Institute for Children's English, National Changhua \\ University of Education \\ laflkuo@cc.ncue.edu.tw
}

\begin{abstract}
This study investigates the incorporation of peer-to-peer ( $\mathrm{P} 2 \mathrm{P})$ computing model into smartphone, and discusses its potential benefits for collaborative mobile users. The goal of this study is to establish a P2P computing environment for network-capable smartphone. Besides, in order to support the interoperability of smartphones and allow them to share information seamlessly, this study proposes a service oriented P2P framework, utilizing $\mathrm{XML}$ and SOAP as the underlying communication media. A prototyping system of the proposed system is implemented. Its implications and applications are also discussed.
\end{abstract}

\section{Introduction}

Smartphone is a mobile device with the voice communication capability of cellular phone and the PIM (Personal Information Management) functionality of PDA. Its slim foam factor enables it to be carried around easily and its computing power and PIM capability allow businessmen to continue their work while on the run. The proliferation of smartphone is a crucial component of the anticipated development of pervasive computing. Experts predict that for smartphones to emerge into the pervasive computing environment they must be ease of use and must provide practical solutions to people's everyday problems [1].The wireless communication technology has also advanced at an unprecedented speed. The bandwidth of WLAN has gone up from $802.11 \mathrm{~b} 11 \mathrm{M}$ bps to $802.11 \mathrm{a} 55 \mathrm{M}$ bps and many PDAs have built-in $802.11 \mathrm{~b}$ WLAN network card. Furthermore, from GSM to GPRS, and now WCDMA, the bandwidth of cellular network has risen up from $9.6 \mathrm{kbps}$ to $2 \mathrm{M}$ bps. Smartphones, with right networking device and access points of Bluetooth, WLAN, and WCDMA networks, are able to connect to the network and interact with other computing devices anytime, anyplace. This creates a dynamic, decentralized, ubiquitous environment where smartphones are able to share services with nearby devices after they have been discovered and they have established relationships with smartphones [2].

$\uparrow$ This work is sponsored by National Science Council, Taiwan (NSC93-2515-S-018-006 \& NSC94-2520-S-018-002). 
The goal of pervasive computing environment is to provide a dynamic, active space where a spectrum of smartphones can freely communicate with each other and share resources. Through the seamless integration of heterogeneous smartphones, new functionality can be created, user productivity can be enhanced, human thought and activity with digital information can be augmented, and the overall daily tasks can be simplified [3]. In short, pervasive computing aims to unobtrusively assist work or recreational activities with information technology that optimizes the environment for people's needs no matter they are at home, office, or public spaces [4]. The current state of the art of pervasive computing is still in its infant stage, facing issues of providing effective supports to enhance the resources sharing and collaborative work for smartphones.

In addition to the advances on mobile devices and wireless networks, the increase in computing power and storage capability of computers has offered peer-to-peer distributed model a new application area. The widespread use of Napster and Gnutella [5] has caused Peer-to-Peer (P2P) computing model to gain the attention of many researchers and companies alike. Most P2P researches focus on how to effectively lookup and utilize the resources in wired network or how to increase the performance of P2P model. However, issues regarding the interoperability of smartphones, the acquiring and sharing of services after being discovered, and the support of collaborative work after being connected in mobile network are not extensively researched.

Fortunately, the emergence of Web services comes to rescue. Web services have been proposed by the $\mathrm{W} 3 \mathrm{C}$ as the standard mechanisms for web applications to communicate and exchange information. The power of Web services comes from the interoperability and extensibility of XML. Through the standard programmatic interfaces, Web services can be combined in a loosely coupled way to achieve complex operations. Sophisticated value-added services can be delivered through the interaction of programs providing Web services [6,7]. This paper proposes a Web services based P2P collaborative framework to support the development of collaborative mobile applications for smarphones. By examining the architectural models of Web services and P2P, and the structural requirements of collaborative mobile applications, the proposed framework provides collaborative Web services and $\mathrm{P} 2 \mathrm{P}$ facilities to allow smartphones to connect to each other dynamically and to share information seamlessly.

\section{Smartphones, P2P, and Web Services Model}

In post $\mathrm{PC}$ (Personal Computer) era, the trend of computing device moves towards small form-factor, light, and portable devices with faster processing speed and network connection ability. This is evident from the widespread use of diverse PDAs and lately smartphones. Figure 1 shows some of the most popular commercial smartphones and their features. 


\begin{tabular}{|l|c|c|c|}
\hline Model: & Palm Tre0600 & SonyEricsson P900 & Motorola MPx \\
\hline OS: & Plam OS 5.2 & Symbian EPOC 7.0 & Mobile Windows \\
\hline CPU: & ARM 144 MHz & ARM 156 MHz & ARM 200 MHz \\
\hline Mem: & $32 \mathrm{M}+$ SD/MMC & $13 \mathrm{M}+$ SD & $32 \mathrm{M}+$ SD/MMC \\
\hline Screen: & $320 \times 320$ pixels & $208 \times 320$ pixels & $240 \times 320$ pixels \\
\hline Net: & CDMA,GRPS, Bluetooth & CDMA, GRPS, Bluetooth & $\begin{array}{c}\text { CDMA, GRPS, } \\
\text { Bluetooth, WiFi }\end{array}$ \\
\hline MM: & EMS, MMS & EMS, MMS & EMS, MMS \\
\hline
\end{tabular}

Fig. 1. Comparisons of three popular smartphones

\subsection{Smartphones}

The characterisitcs of smartphones making them ideal devices for collaborative mobile applications are:

a. Network-ready: Most smartphones have at least one data communication chipset such as Bluetooth, GPRS or WCDMA.

b. Mobility: With wireless network and smartphones, we are moving from an infrastructure of tethered computing and communications toward mobility. That is, smartphones enable us to work even when we are on the move.

c. Multimedia: Smartphones are capable of recording/playing audio/video and playing mobile games in addition to the MMS.

d. Virtuality: The software environment brought by smartphones is similar in functionality to their desktop counterpart to allow people to work whenever they desire and wherever they are.

\subsection{P2P Computing Model}

P2P was said to be the third network revolution since the invention of Mosaic Web browser. P2P is a communication model in which each party has the same capabilities and either party can initiate a communication session. In most cases, a node in P2P model can act both as a client and as a server depending on whether it acts as a requester or a servant.

P2P can be implemented in hardware as the IBM's APPN (Advanced Peer-to-Peer Networking) that supporting the P2P communication model. Or it can be implemented as a software solution as the likes of Napster where a group of computer users with the same networking program connect with each other and directly access files from one another's hard drives. Contrasting to the P2P is the client/server model where a client makes a service request from a server which fulfills the request of a client. Typically, the server's computing power is far superior to the client.

\subsection{Web Services Model}

Nowadays, as more and more companies seek to conduct business over the Internet, the problem they face is how to make their applications work with those of their 
customers and suppliers. With proprietary message format, companies can get their applications to talk to each other. However, as the number of applications goes up, the number of possible communication paths increase dramatically. One solution for this problem is to use middleware as the communication mediator but it does not scale well to large applications.

Web services are applications that adopt a universal language to send data and instructions over the Internet to one another. The goal of Web services is to provide a flexible and cost-effective solution for enterprises conducting business on the Internet. Web services are open, distributed software components, and are built on top of the standards of XML, SOAP, WSDL (Web Services Description Language), and UDDI (Universal Description Discovery and Integration) [8,9]. Programmers can use their favorite programming language and operating system to describe and write Web services which can be invoked over the Web. Please see [10] for detailed descriptions of Web services.

\section{The Framework and Implementation}

To achieve the interoperability of mobile devices, this study proposes the Web services based service framework utilizing SOAP as the underlying message format to communicate with mobile devices of different platforms.

Figure 2 shows the conceptual diagram of the proposed framework. Through the common interface of Web services module and the P2P mechanism, every mobile device can access the Web services offered by any locatable mobile device. This framework is particularly suitable for dynamic, ad hoc wireless network. But how does the Web services and P2P work in this environment? How does one mobile device know the status of the other in $\mathrm{P} 2 \mathrm{P}$ way? To solve these problems, we design mechanisms which allow each mobile device to register its services. After a service provider has written and deployed its services, it registers them to the UDDI registry server, implemented as a SOAP server. Then, a service requester queries the SOAP directory server to see if there exists any service meeting its requirement. If there exists one, the SOAP server forwards the service provider's WSDL to the service requester to allow them to communicate with each other directly without the need of the SOAP server afterwards. This enables the P2P communication between the service requester and the service provider. Figure 3 shows the P2P communication process between two mobile devices. Notice that every mobile device can act as a client (service requester) or a server (service provider) depending on its role.

The proposed framework has the following characteristics:

- Web services: The services offered by a mobile device are encapsulated into Web services, facilitating a uniform communication path among peer mobile devices.

- SOAP-based message: The delivery of message is based on SOAP. Thus, applications written in different programming languages and run on different operating systems can still communicate with each other to achieve the interoperability.

- Service transparency: The location of a service provider is of no concern to a service requester since the binding between them occurs automatically. The selection of a service provider is done in server side and its selection criteria might 
be distance or time, depending on the algorithm used by the server.

- Peer-to-Peer: Every mobile device is treated equally as a peer. Everyone has the ability to provide services for others and to use the services from others. This is important for mobile devices because (1) resources in every mobile device are scarce, and (2) performance of mobile devices can be maximized while the load of the server can be minimized.

- Ubiquitous service: Services might not exist when requested but the requester will be notified whenever they appear. This is achieved through UDDI. It eliminates the need of the service requester to continuously poll the server for the service.

- Extensible: The functionalities of applications can be extended by just adding new Web services modules and registering them onto the UDDI server. The programming effort is reduced since the database connection and P2P communication complexity are encapsulated in Web services modules, hidden from applications.

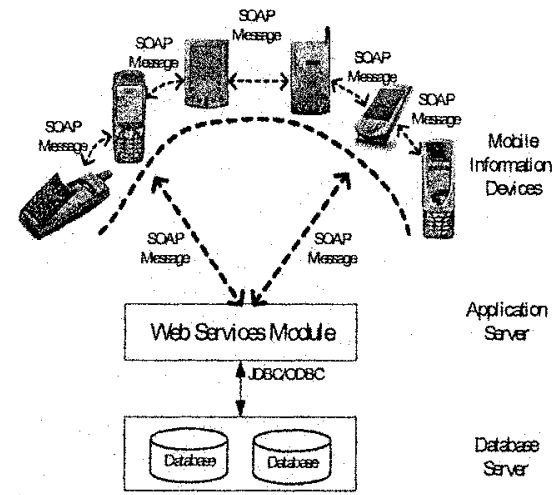

Fig. 2. The conceptual diagram of the proposed framework

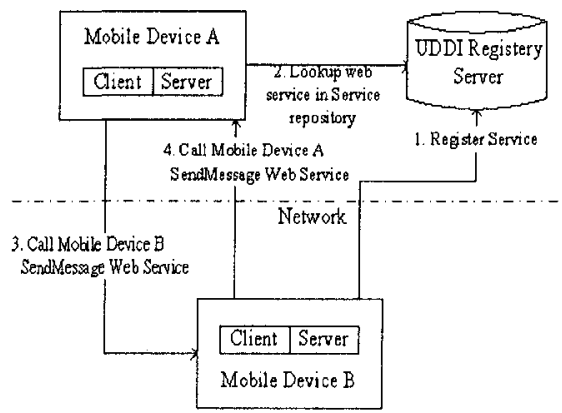

Fig. 3. The P2P communication process between two mobile devices 


\subsection{Layered Design}

The design of the proposed framework is based on layered architecture, as shown in Figure 4 . In this approach, each layer supports certain functionality which is used by its immediate upper layer.

The proposed framework consists of three layers. The system library layer consists of parsers for parsing XML, SOAP, WSDL, and UDDI messages. The P2P service layer is designed to facilitate the registration, indexing, advertisement, discovery, and notification of services of mobile devices. Collaborative mobile services and multimedia mobile services belong to the application services offered by mobile devices and are encapsulated into Web services. Examples of collaborative mobile services include file sharing, scheduling, instant messaging, and task arrangement. Multimedia mobile services include audio/video messaging, graphics drawing, and gaming.

The advantages of this approach include:

- Simplified design: By dividing functions to each layer, it is much easier to design and implement the framework since one only has to worry about implementing some functionalities for each layer. Besides, flaws are easier to track.

- Adaptability: If a better algorithm is going to replace an old one in a certain layer, other layers will not be affected as long as interfaces between them stay the same.

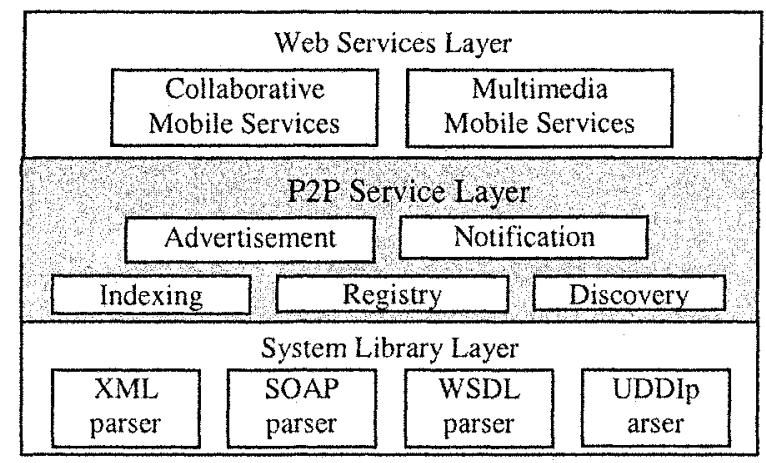

Fig. 4. Layered design of the proposed framework

\subsection{Class Diagram}

Figure 5 shows the proposed framework's top level class diagram which is composed of seven classes: MobIntMsg (Mobile Instant Messaging), SrvSptWS (Service Support Web Services), MobInfoShr (Mobile Information Sharing), MobGrpInfoMgt (Mobile Group Information Management), ContactWS (Contacts Web Service), TaskWS (Tasks Web Service), and ApptWS (Appointments Web Service) ${ }^{\ddagger}$.

\footnotetext{
$\$$ Due to space limit, other classes are omitted here.
} 


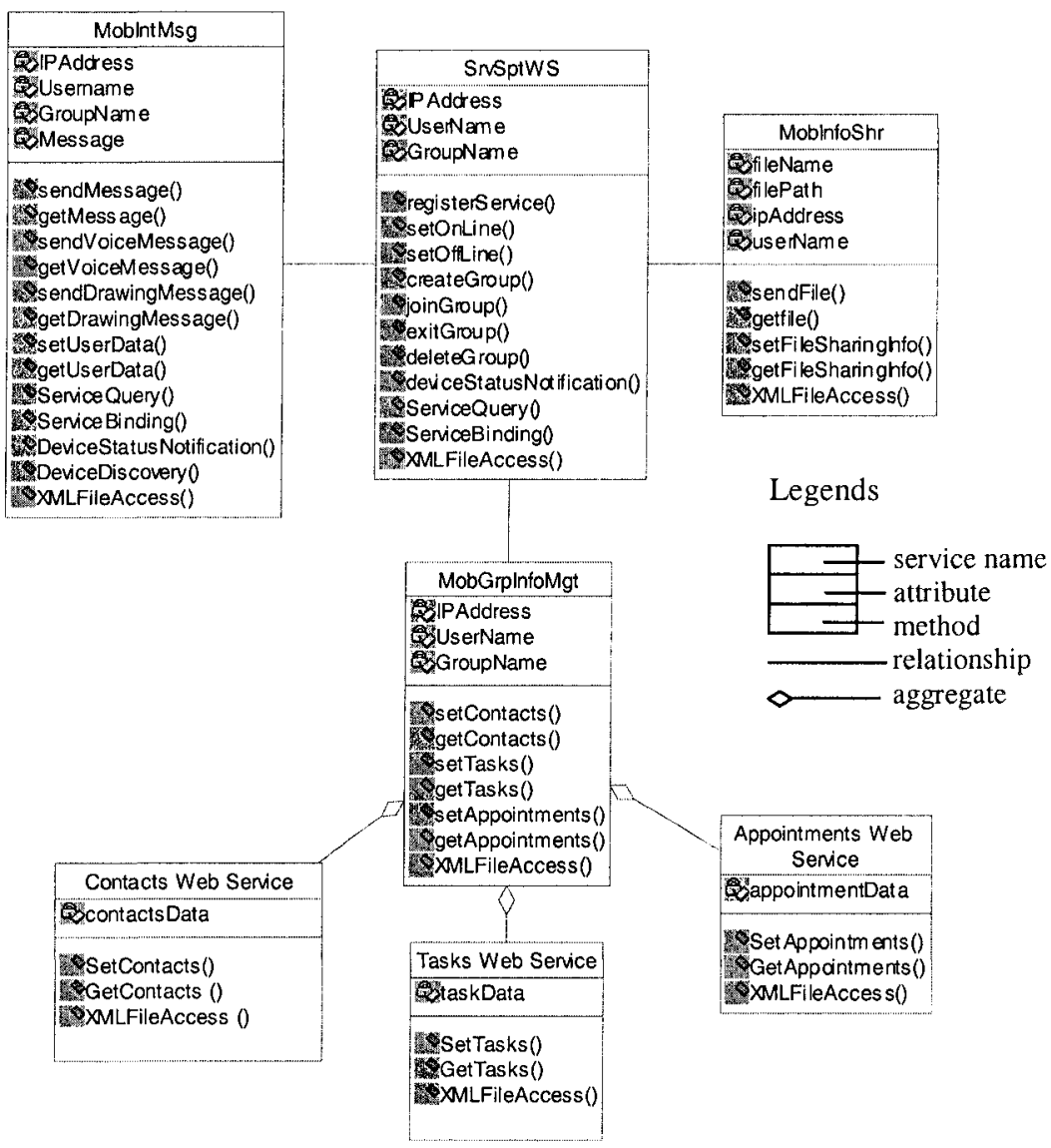

Fig. 5. Class diagram of Web services based P2P collaborative framework

\subsection{Implementation}

The proposed framework was implemented using Java programming languages. Two personal computers are setup as the UDDI register application server and SOAP application server. The UDDI register application server was implemented using IBM's UDDI service registry and the SOAP application server was built on top of Apache SOAP server. All Web services were implemented using Java and Sun Microsystems's J2EE SDK. Three Compaq iPAQ H3870 with D-Link $802.1 \mathrm{lb}$ WLAN were used as test beds for smartphones. 


\subsubsection{XML Configuration and SOAP Message Format}

In order for the Web services based P2P collaborative framework for smartphones to work, there must be a mechanism for smartphones to know each other, to understand the capability of each other, and to communicate with each other. All devices related information is stored in a configuration file. Figure 6 shows an XML example of the status information of an iPAQ 3870 PDA with WLAN card. Other configuration files (not shown here) include user information, capability information, directory information, resource information, etc.

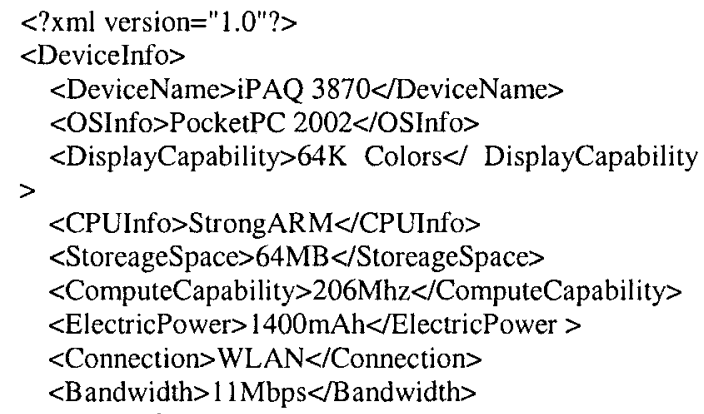

$<!-$ device infor, -->

$<!-$ model -->

$<!--$ OS -->

$<!-$ screen -->

$<!-$ CPU -->

$<!--$ memory-->

$<$ !- computing power -->

$<!--$ electric power $->$

$<$ !- network type -->

$<$ !- bandwidth $\rightarrow>$

Fig. 6. A device configuration expressed in XML

\subsubsection{SOAP Message Format}

Web services, based on the SOAP message, serve as the communication media. SOAP message is composed of Envelope, Header, and Body elements where Header and Body elements are the sub-elements of Envelope. Header contains the information of message arguments while Body contains remote methods definition and arguments. Figure 7 shows a file sharing SOAP message showing the device owner is Sam Chiang with IP 163.23.199.124 and its file directory information.

<FileSharingInfo xmlns:m='urn:Foo'>

<Owner ip="163.23.199.124" name="Sam Chiang"/>

$<$ SharingInfo>

$<$ DIR name="IMY Documents" $/>$

$<$ FILE name="msg. wav" size $=" 2522 " /><$ FILE name="j531.jpg" size="76195"/>

$\cdots$

$<$ DIR name="|MyMusic" $>$

$<$ FILE name="Bon Jovi.mp3" size="6671"/>

$<$ DIR $>$

$</$ SharingInfo $>$

$<$ FileSharingInfo $>$

Fig. 7. A file sharing SOAP message 


\section{A Collaborative Mobile Application Scenario}

As a concept proof of the proposed Web services based P2P collaborative framework, a collaborative mobile application for smartphones is built on top of the framework. The setup is as followings: one client running on top of iPAQ H3870 was implemented using eVB while another client running on another iPAQ H3870 was implemented using Java (using Jeode JVM). All servers are implemented by Java using Apache Tomcat, Sun Microsystems J2EE, and IBM's UDDI SDK.

Figure 8 shows some interfaces of this collaborative applications. Figure 8a offers users and group management with functions of creating, adding, modifying, or deleting users or groups. Figure $8 \mathrm{~b}$ shows a collaborative scheduling of a group of people. If there is no conflict among all people participated, then a new schedule is created and set. If there is a conflict, events are triggered to notify people with conflicting schedule. Figure $8 \mathrm{c}$ shows file sharing service which provides efficient file transfer with auto-reconnection and auto-retransmission. Figure 8d shows ink message service allowing people to communicate with each other using hand written script. Other services include answering machine service, real-time photo taking and transmitting service, Goolge search engine Web service, and so on.

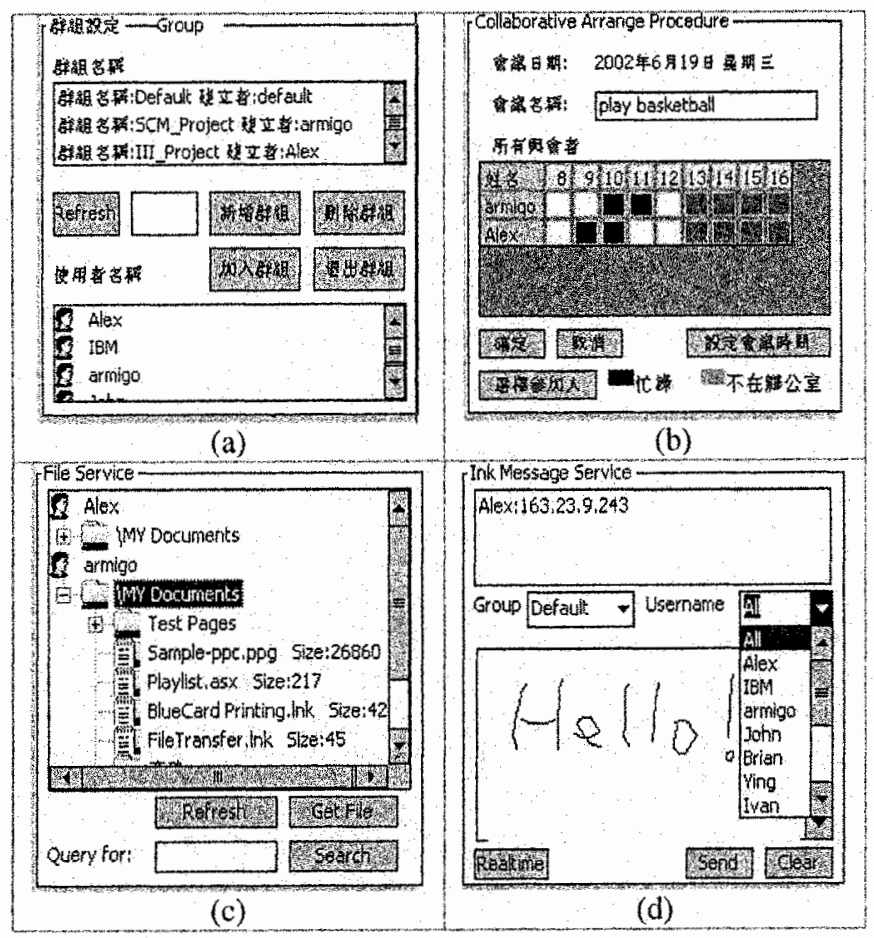

Fig. 8. Some interfaces of a collaborative application

\footnotetext{
$\$$ The snapshots of interfaces are taken from PocketPC simulator for clarity.
} 


\section{Conclusion}

Because of the rapid development of computer and Internet technologies, the trend towards the integration of wired network, wireless network, and mobile devices is inevitable. Smartphones, with right networking device and access points of Bluetooth, WLAN, and WCDMA networks, are able to connect to the network and interact with other computing devices anytime, anyplace. The authors envision interoperability among smartphones or mobile computing devices will be a critical issue of pervasive computing.

By examining the architectural models of Web services and P2P, and the structural requirements of collaborative mobile applications, this study built a Web services based $\mathrm{P} 2 \mathrm{P}$ collaborative framework providing collaborative Web services and $\mathrm{P} 2 \mathrm{P}$ facilities to allow smartphones to connect to each other dynamically and to share information seamlessly. As a proof of concept, a collaborative application based on the proposed framework was built where two iPAQ H3870 clients implmented using Java and eVB were ablie to communicate and share information with each other through Web services and P2P. Future work includes incorporating the location information of mobile users to provide a location-aware collaborative P2P framework; providing support for audio/video streaming to enable a realtime video conferencing; adding support for $\mathrm{J} 2 \mathrm{ME}$ platform which has been popular lately.

\section{References}

1. Pierre, S.: Mobile Computing and Ubiquitous Networking: Concepts, Technologies and Challenges. Telematics and Informatics. 18 (2001) 109-131

2. Kortuem, G.: When Peer-to-Peer Comes Face-to-Face: Collaborative Peer-to-Peer Computing in Mobile Ad-Hoc Networks. Proceedings of the 2001 International Conference on Peer-to-Peer Computing. (2001) 27-29

3. Friday, A., Davies, N., Wallbank, N, Catterall, E., Pink, Stephen: Supporting Service Discovery, Querying and Interaction in Ubiquitous Computing Environments. 10 (2004) 631-641

4. Roman, M.: Gaia: A Middleware Infrastructure to Enable Active Spaces. IEEE Pervasive Computing. 1:4 (2002) 74-83

5. Stoica, I.: Chord: A Scalable Peer-To-Peer Lookup Protocol for Internet Applications. IEEE/ACM Transactions on Networking. 11:1 (2003) 149-160

6. Sutherland, J., Heuvel, J. V. D.: Enterprise Application Integration and Complex Adaptive Systems. Communications of the ACM. 45:10 (2002) 59-64.

7. Mougin, P., Barriolade, C.: Web Services, Business Objects and Component Models. Orchestra Networks, NY (2001)

8. Jopsen, T.: SOAP Cleans Up Interoperability Problems on the Web. IT Professional. 3:1 (2001) $52-55$

9. IBM UDDI Service Registry. hutp://www-3.bim.com/services/uddi/protect/find (2004)

10. Kao, J.: Developer's Guide to Building XML-based Web Services with the Java 2 Platform, Enterprise Edition. Sun Microsystems, CA (2001). 